\title{
ANÁLISE EXPERIMENTAL E CUSTOS DE TELHADOS VERDES COMERCIAIS E FABRICADOS COM GARRAFAS PET PARA REDUÇÃO DE CHEIAS URBANAS NA AMAZÔNIA
}

\section{EXPERIMENTAL ANALYSIS AND COSTS OF COMMERCIAL GREEN ROOFS AND MADE WITH PET BOTTLES TO REDUCE URBAN FLOODS IN THE AMAZON}

\author{
EDKEYSE DIAS GONÇALVES | UFPA \\ CLAUDIO JOSÉ CAVALCANTE BLANCO, PhD. | UFPA \\ VANESSA DA ROSA WATRIN, M.SC.|UFPA \\ CARLOS EDUARDO AGUIAR DE SOUZA COSTA, M.SC.| UFPA
}

\begin{abstract}
RESUMO
A impermeabilização do solo proveniente da urbanização interfere diretamente no ciclo hidrológico, pois o cenário antes coberto pela vegetação dá espaço ao cenário impermeabilizado do asfalto e do concreto. Isso provoca redução na infiltração da água precipitada, acarretando o aumento do escoamento superficial e inundações. O objetivo do trabalho foi comparar experimentalmente o desempenho hidrológico e os custos de um telhado verde de politereftalato de etileno - PET (TV I) com um telhado verde comercial (TV II), usando um telhado de fibrocimento como padrão. O desempenho do TV I foi ligeiramente superior ao desempenho do TV II quanto ao retardo do escoamento superficial. Somado a isso, o custo do telhado construído com PET é menor que o custo do telhado verde comercial, desconsiderando-se os custos com fabricação e montagem dos telhados verdes. Nesse contexto, é possível afirmar que o uso de telhados verdes pode contribuir para a redução de cheias; e que o uso de materiais recicláveis na construção de telhados verdes mostra-se como uma alternativa viável tanto ambientalmente como economicamente.
\end{abstract}

PALAVRAS CHAVE: CN de telhados verdes; Redução da vazão de pico; Material reciclável

\begin{abstract}
The waterproofing of the soil coming from the urbanization interferes directly in the hydrological cycle, since the scenario previously covered by the vegetation gives space to the waterproofed scenery of the asphalt and the concrete. This causes a reduction in the infiltration of precipitated water, leading to increased runoff and flooding. The objective of this work was to compare experimentally the hydrological performance and costs of green roof of polyethylene terephthalate - PET (TVI) with commercial green roof (TV II) using asbestos cement roof as standard. The performance of TV I was slightly higher than the performance of TV II for the delay of the runoff. In addition to this, the cost of the roof constructed with PET is less than the cost of the commercial green roof, disregarding the costs of manufacturing and assembling the green roofs. In this context, it can be stated that the green roof can contribute significantly to the reduction of floods and that the use of recyclable materials in the construction of green roofs proves to be a viable alternative both environmentally and economically.
\end{abstract}

KEY WORDS: CN of green roofs; Peak flow reduction; Recyclable material 


\section{INTRODUÇÃO}

Os processos de industrialização e urbanização têm provocado diversas alterações sobre o ambiente natural. Os espaços outrora cobertos pela vegetação abrem lugar a cenários impermeabilizados por asfalto e concreto. Esta drástica mudança compromete diretamente a qualidade de vida das pessoas nas cidades, deixando o ambiente urbano propício a enchentes entre outros problemas ambientais, como por exemplo: poluição visual, poluição do ar, poluição das águas e a intensificação do aumento de temperatura urbana com as "ilhas de calor" (FRANÇA, 2012). A impermeabilização proveniente da urbanização desordenada interfere diretamente no ciclo hidrológico, modificando o comportamento do escoamento superficial, da infiltração e o desabastecimento das águas subterrâneas, além de diminuir a evapotranspiração (TARGA et al., 2012). De acordo com Justino et al. (2011), o acréscimo de $54 \%$ de área impermeável pode provocar um aumento na vazão de pico em até $59,40 \%$. Tal fato justifica o aumento da ocorrência de inundações em centros urbanos, sendo necessário o uso de medidas e alternativas sustentáveis que ajudem a minimizar o impacto ambiental causado pela impermeabilização urbana.

Diante desses problemas provocados pela urbanização, uma nova abordagem de proteção ao ambiente urbano levanta a ideia do Desenvolvimento Urbano de Baixo Impacto ou Low Impact Development - LID, tendo como princípio a conservação e preservação da cobertura vegetal urbana e a execução de projetos de engenharia que respeitem as peculiaridades locais naturais, assegurando a conservação qualitativa e quantitativa de processos hidrológicos (SOUZA et al.; 2012). Assim, o mecanismo LID destaca como dispositivos de controle de águas pluviais a construção de cisternas, pavimentos permeáveis e telhados verdes (TVs). Karteris et al. (2016), Razzaghmanesh et al. (2016), Huang et al. (2016) também destacam, entre os sistemas disponíveis no campo da construção civil sustentável e bioclimática, o Telhado Verde (TV), e o apresentam como uma técnica de controle do escoamento superficial e isolamento térmico, com o objetivo de reduzir inundações e ilhas de calor em meio urbano.

É importante que os telhados verdes sejam introduzidos na indústria da construção civil como uma abordagem ecológica para o ambiente construído sustentável, mas o alto investimento inicial com estruturas verdes ainda limita o seu uso em grandes centros urbanos de diversos locais do mundo (BERARDI et al., 2014). Segundo Bianchini e Hewage (2012), uma boa opção para reduzir o custo de instalação de telhados verdes é a reutilização e reciclagem de materiais de polietileno no mercado da construção civil sustentável, o que ajudaria a reduzir os custos e o peso total do telhado, bem como melhorar o desempenho das camadas sem comprometer os benefícios dos telhados verdes. Vila et al. (2011), Pérez et al. (2012), Rincón et al. (2014), Chenani, Lehvävirta e Häkkinen (2015) destacam a necessidade de avaliar o impacto ambiental dos materiais no processo de construção de um telhado verde, tendo em vista que dentro do ciclo de vida de um material deve-se levar em consideração a quantidade de matéria prima utilizada, o transporte, o processo de produção e a energia empregada neste processo.

Belém, capital do Pará, passa por forte crescimento populacional desde a década de 1960, o que ocasionou a construção de um espaço urbano impermeabilizado e desordenado, modificando o ciclo hidrológico urbano da cidade. Segundo Tavares (2009), Belém não tem um sistema de drenagem eficiente, o que intensifica os alagamentos durante os eventos de chuva extremos, os quais associados à maré alta causam grandes prejuízos aos moradores. Assim, diante do contexto exposto, foram testados experimentalmente dois tipos de telhados verdes em clima amazônico, um comercial e outro fabricado com garrafas PET, permitindo avaliar a redução da vazão de pico de cheias para a redução do escoamento superficial e custos desses telhados quando comparados a um telhado convencional de fibrocimento.

\section{MATERIAL E MÉTODOS}

\subsection{Localização doTelhado VerdeExperimental} O telhado verde experimental está localizado no Campus da Universidade Federal do Pará (Figura 1), às margens do rio Guamá, aproximadamente $120 \mathrm{~km}$ do Oceano Atlântico. Segundo a classificação de Köppen et al. (1936), o clima da Região Metropolitana de Belém (RMB) é do tipo "Am", quente e úmido, contendo pouca variação de temperatura e umidade ao longo do ano. Entretanto, os totais pluviométricos dos primeiros seis meses são maiores que os dos últimos seis meses.

\subsection{Construção do Telhado Verde Experimental}

O protótipo foi dividido em três lotes. Dois lotes com $1 \mathrm{~m} 2$ de área com cobertura verde, um lote posicionado entre os dois, com telhado convencional de telha de fibrocimento também com $1 \mathrm{~m} 2$ de área. O telhado verde do tipo I (TV I) com dimensão de $1 \times 1 \times 0,1 \mathrm{~m}$ foi construído com camada de drenagem oriunda de material reutilizado (Polietileno Tereftalato - PET) sobre telhas de fibrocimento impermeabilizadas (1), camada filtrante (2), substrato (3) e vegetação (4). As camadas do TV I são detalhadas na Figura 2. 

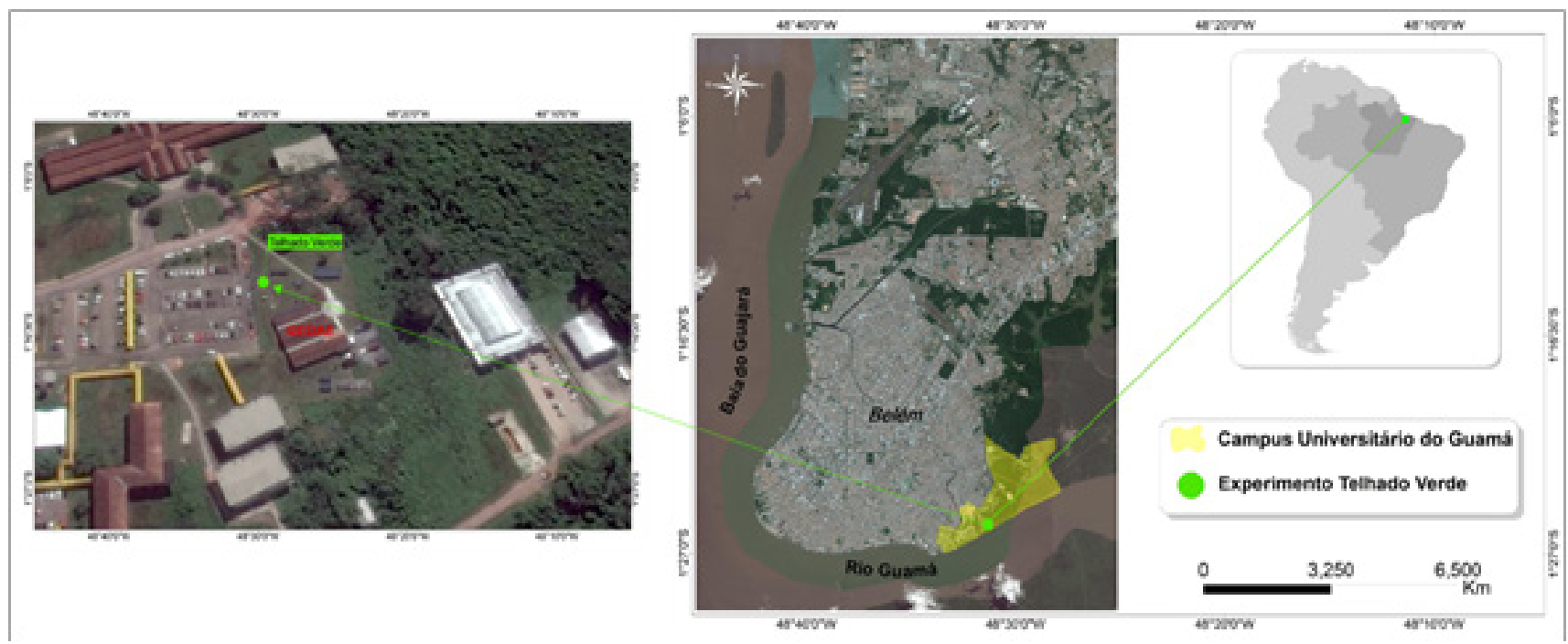

Figura 1 - Localização do Telhado Verde Experimental

Fonte: Autores
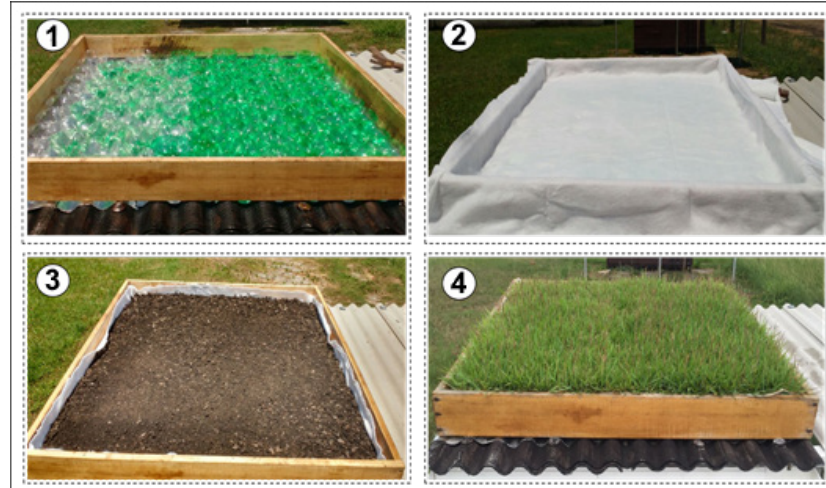

Figura 2 - Montagem do telhado verde tipo I Fonte: Autores

Sendo, (1) camada de drenagem fabricada a partir de fundos de garrafas PET arranjadas lado a lado, o quantitativo necessário foi de 100 fundos de garrafas para $1 \mathrm{~m} 2$ de telhado, com espessura de $4 \mathrm{~cm}$; (2) camada filtrante, também conhecida como Manta Geotêxtil (composta 100\% de poliéster de cor branca modelada com dimensões de 1,20 x 1,20 m; (3) camada de substrato comercial denominada "Flores \& Folhagens" da BIOMIX®. Nesse caso, trata-se de um substrato que concentra macros e micros nutrientes essenciais à vida vegetal, ou seja, turfa, casca de pinos, vermiculita, resíduo orgânico classe $A$, serragem e esterco compostado. E (4) Camada de vegetação representada pela Grama Esmeralda (Zoysia japonica Steud).

O Telhado verde do tipo II (TV II) com as mesmas dimensões do TV I, é composto de módulos drenantes pré-fabricados, denominados camada de drenagem Alveolar Leve. Possui formato retangular com dimensões de $0,14 \mathrm{x}$ $11,5 \times 0,35 \mathrm{~cm}$ (Espessura, Largura e Comprimento), conforme Figura 3.
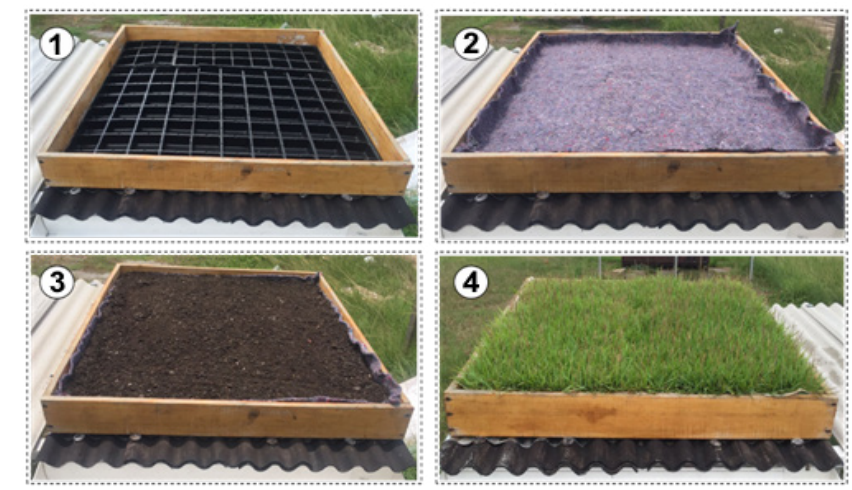

Figura 3 - Montagem do telhado verde tipo II Fonte: Autores

Sendo, (1) camada de drenagem Alveolar Leve sobre telhas impermeabilizadas; (2) camada filtrante sobre a camada de drenagem Alveolar Leve; (3) camada de substrato sobre a camada filtrante e camada de drenagem Alveolar Leve; $\mathrm{E}$ (4) camada de vegetação sobre o sistema de camada de drenagem Alveolar Leve. Na Figura 4 é apresentada a bancada experimental com os três telhados na sequência: TV I, telhado convencional (TC) e TV II. TV I e TV II são classificados como telhados verdes extensivos, pois a espessura total deles é de $10 \mathrm{~cm}$.

Como mostra a Figura 4, os dados foram monitorados por meio de registros de câmeras filmadoras, as quais gravaram os eventos de chuva. O escoamento excedente é o resultado da chuva total incidente, exceto a parcela da chuva que fica retida no telhado verde, por meio da interceptação da água pela grama, do armazenamento de água pelo substrato e da camada de drenagem. Assim, o escoamento excedente é conduzido por meio de caIhas interligadas por tubos de PVC, que desaguam nos 
reservatórios com capacidade de armazenamento de 50 L. Esses reservatórios possuem réguas com divisões em centímetros, permitindo medir a lâmina de água escoada. Com os dados monitorados, foram determinados hidrogramas de projeto com vazões medidas a cada 5 min.

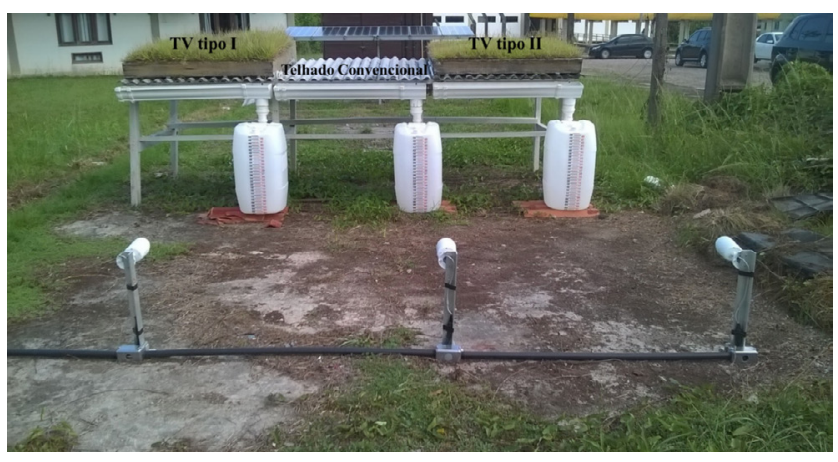

Figura 4 - Vista frontal dos três telhados verdes com monitoramento por câmeras Fonte: Autores

\subsection{Capacidade Máxima de Armazenamento de Água dos Telhados Verdes}

Segundo Ohnuma Jr. (2014), a capacidade de armazenamento de água em telhados verdes pode variar com a intensidade da chuva e com a umidade antecedente do solo. Assim, o armazenamento de água em telhados verdes (S) pode ser calculado a partir da altura da platibanda sobre o telhado (h) e da porosidade do solo (Øs) utilizado no plantio (Equação 1)

$$
\boldsymbol{S}=\boldsymbol{h} \cdot \boldsymbol{\varnothing} \boldsymbol{s}
$$

Sendo $S(\mathrm{~mm})$ o armazenamento máximo de água acumulada no telhado verde; $\mathrm{h}(\mathrm{mm})$ a altura da platibanda sobre o telhado verde; e $\emptyset$ s (\%) a porosidade do solo utilizado no plantio.

A determinação de S é necessária para o cálculo de CN (Equação 2) através da metodologia de NRCS (2007).

$$
C N=25400 /(S+254)
$$

O método CN (Curva-Número) é o parâmetro que relaciona o tipo de solo com sua capacidade de drenagem. $\mathrm{O}$ valor de $\mathrm{CN}$, que é um parâmetro adimensional, varia em uma escala de 1 a 100. Portanto, quanto mais impermeável for o solo, maior o valor de CN e menor será a retenção da chuva pelo solo (CUNHA et al.,2015).

Outro parâmetro importante é a condição de umidade antecedente do solo (AMC), pois o armazenamento de água no telhado verde pode apresentar variações em um determinado evento de chuva e é sabido que a eficiência do telhado verde está diretamente relacionada à umidade presente no substrato resultante dos eventos de chuvas anteriores (TASSI et al., 2014). Assim, para se verificar a eficiência do telhado verde, deve-se considerar a influência da AMC. Neste trabalho foi utilizada a classificação estabelecida por Tucci (2005), que avalia o volume de chuva para 5 dias que antecedem o evento chuvoso monitorado, dividindo a AMC em três diferentes classes. AMC I: situação em que os solos estão secos, considerando que a precipitação acumulada dos cinco dias anteriores é menor que $13 \mathrm{~mm}$; AMC II: situação média em que os solos correspondem à umidade da capacidade de campo. A precipitação acumulada dos cinco dias anteriores é maior que $13 \mathrm{~mm}$ e menor que 28 mm; e AMC III: situação em que ocorreram precipitações consideráveis nos cinco dias anteriores e o solo encontra-se saturado. Nesse caso, a precipitação acumulada dos cinco dias anteriores é maior que $28 \mathrm{~mm}$.

\section{RESULTADOS E DISCUSSÃO}

Na Figura 5 são apresentados 8 eventos de chuvas monitorados. A partir dos hidrogramas, foi possível verificar o comportamento hidrológico dos telhados verdes extensivos e convencional. Os dados foram coletados no período de 20/09/2017 a 03/11/2017. Dentre os vinte oito eventos de chuva do período, oito resultaram em hidrogramas (Figura 5), treze não geraram escoamento nos telhados verdes, pois resultaram de chuvas inferiores a $2 \mathrm{~mm}$ e sete eventos foram perdidos por falha no equipamento ou falta de energia elétrica. Os volumes de chuva registrados nos oito eventos válidos variaram de 2,6 a $11 \mathrm{~mm}$, enquanto os escoamentos superficiais dos telhados verdes variaram de 0 a 1,2 L/min. O TV I apresentou saturação média de suas camadas após 16 minutos do início de cada evento de chuva. Em contrapartida, o TV II mostrou suas camadas saturadas após 12 minutos para cada evento de chuva. O tempo médio que o telhado convencional levou para começar a escoar superficialmente foi de 1 minuto e 30 segundos.

Com relação ao aspecto quantitativo, o TV I proporcionou, em 6 dos 8 eventos monitorados, maior amortecimento ou retardo do escoamento superficial comparado ao TV Il e ao telhado convencional. Essa mesma característica foi observada por Costa et al. (2012) para o estado de São Paulo. Nesse caso, os autores demonstraram que a diferença de vazão de pico entre os telhados verdes foi mínima, contudo, o telhado convencional comparado aos telhados verdes apresentou maior vazão de pico, como era esperado.

Com relação à $\mathrm{AMC}$, foi verificado que nos eventos com maior volume de chuva precitado nos cinco dias antecedentes (Tabela 1), os eventos 05 e 06 de classe AMC 3 apresentaram menor porcentagem de retenção que o evento 01 de classe AMC 1, que é o evento de menor volume de chuva antecedente. Se for avaliado o volume de chuva precipitado nos eventos e o volume das chuvas dos cinco dias que 
antecederam os eventos, pode-se inferir que, para esta amostra, a umidade antecedente do solo (AMC) apresentou maior impacto na capacidade de retenção dos telhados verdes do que o volume precipitado. Entretanto, a amostra não incluiu eventos extremos, que segundo Carter e Rasmussen (2006) têm forte influência na redução da capacidade de retenção dos telhados verdes. Assim, a variabilidade da retenção

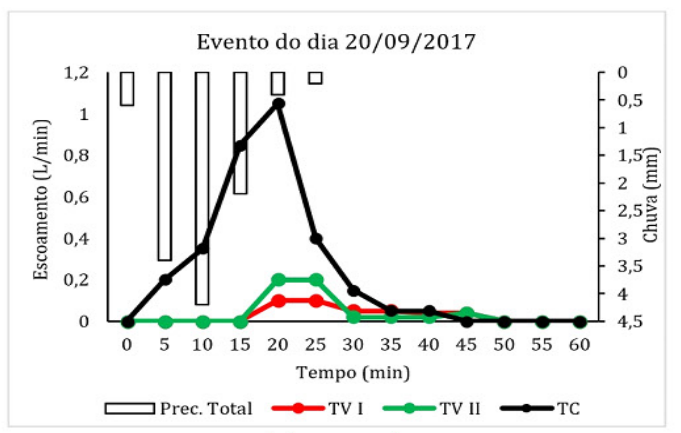

Evento 1

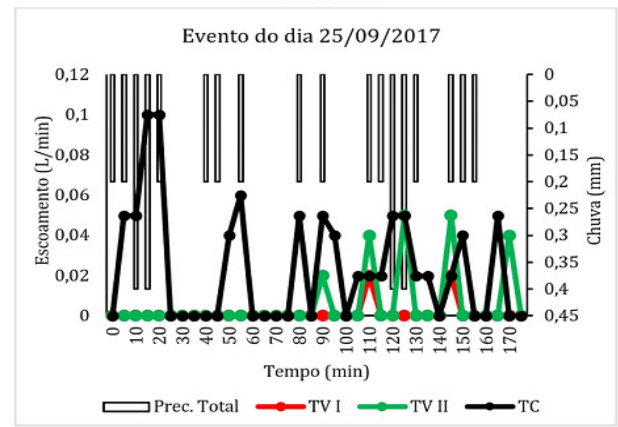

Evento 3

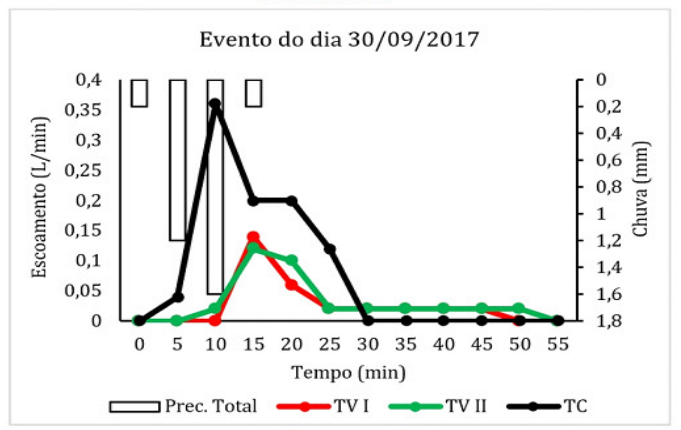

Evento 5

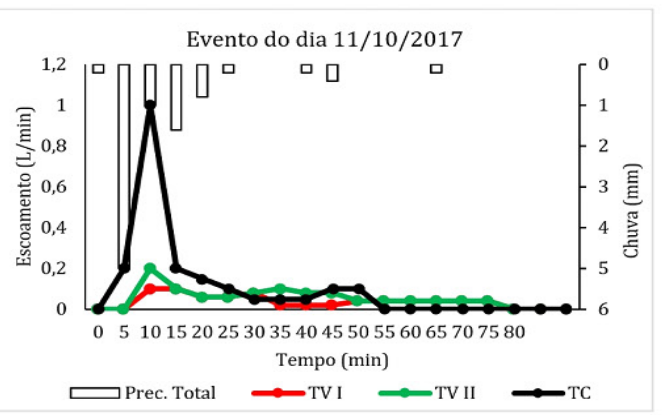

Evento 7 hídrica encontrada nos TV I e TV II para eventos em período mais seco e úmido mostraram-se semelhantes aos estudos de Bacovis e Nagalli (2013), os quais constataram que a capacidade do telhado verde de reter água está diretamente relacionada com a condição inicial de saturação do telhado verde. Tassi et al. (2014) também verificaram que o telhado verde no período chuvoso apresentou menor eficiência.

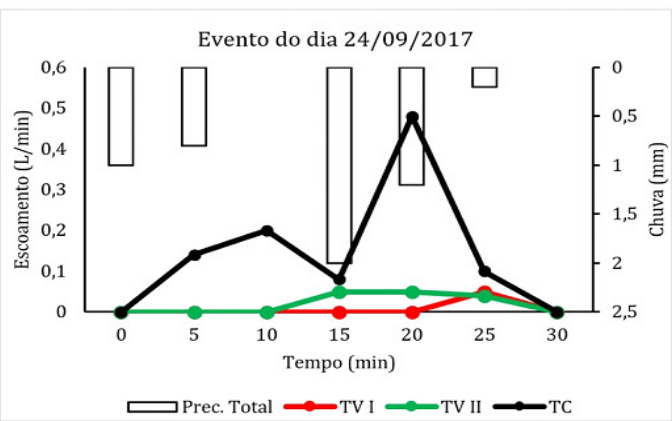

Evento 2

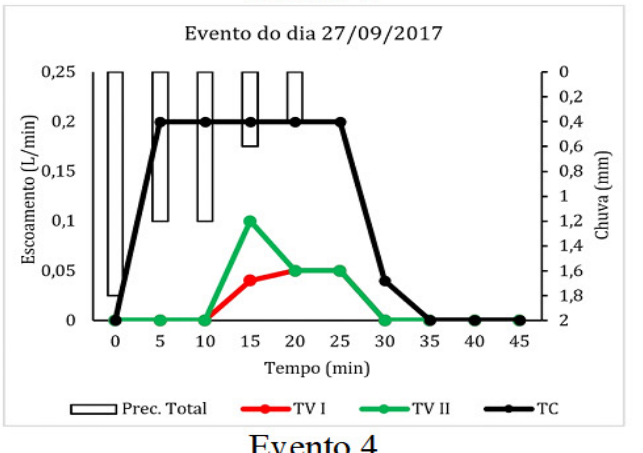

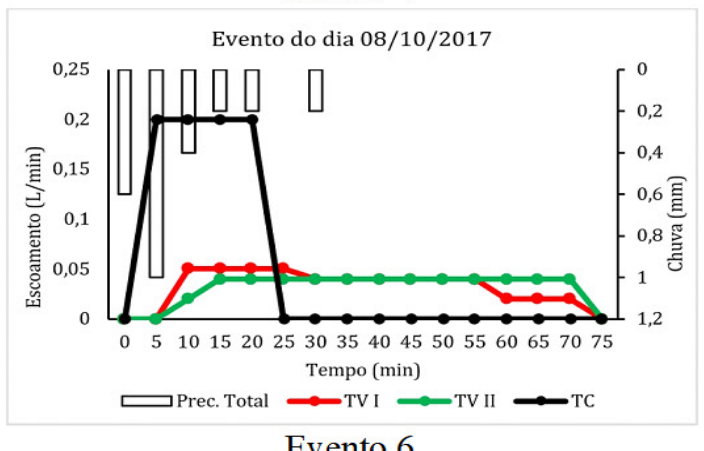

Evento 6

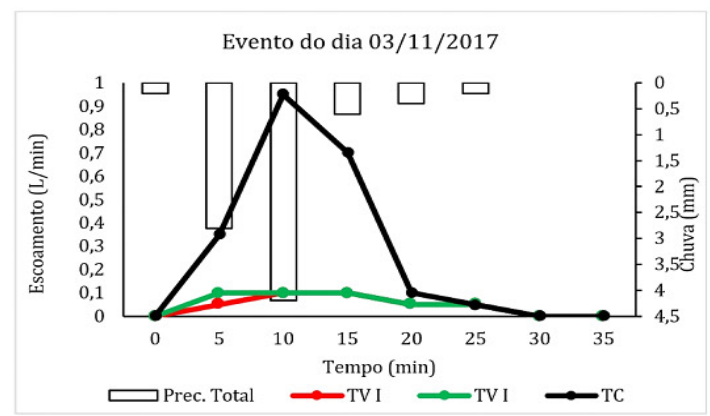

Evento 8

Figura 5 - Hidrogramas dos oito eventos de chuva nos TVI, TV II e TC Fonte: Autores 


\begin{tabular}{|l|l|l|l|l|l|l|}
\hline Evento & Data & Volume dos últimos 5 dias $(\mathbf{m m})$ & AMC & Volume de chuva precipitado $(\mathbf{m m})$ & Retenção TV I & Retenção TV II \\
\hline EV 01 & $20 / 09 / 2017$ & 1,20 & 1 & 11 & $87,74 \%$ & $83,87 \%$ \\
\hline EV 02 & $24 / 09 / 2017$ & 11,4 & 1 & 5,2 & $95,00 \%$ & $86,00 \%$ \\
\hline EV 03 & $25 / 09 / 2017$ & 16,6 & 2 & 4,4 & $95,29 \%$ & $76,47 \%$ \\
\hline EV 04 & $27 / 09 / 2017$ & 9,6 & 1 & 5,2 & $86,54 \%$ & $80,77 \%$ \\
\hline EV 05 & $05 / 10 / 2017$ & 39,8 & 3 & 3,2 & $67,39 \%$ & $60,87 \%$ \\
\hline EV 06 & $08 / 10 / 2017$ & 37,8 & 3 & 2,6 & $37,50 \%$ & $37,50 \%$ \\
\hline EV 07 & $11 / 10 / 2017$ & 11,2 & 1 & 9,4 & $65,00 \%$ & $50,00 \%$ \\
\hline EV 08 & $03 / 11 / 2017$ & 11,8 & 1 & 8,4 & $83,72 \%$ & $81,40 \%$ \\
\hline
\end{tabular}

Tabela 1 - Relação da AMC e do volume precipitado com a retenção Fonte: Autores

Avaliando os percentuais médios da retenção hídrica constata-se que: das precipitações ocorridas, $77,27 \%$ ficaram retidas no TV I; e 69,61\% no TV II. Portanto, o TV I mostra-se mais eficiente que o TV II em até 7,66\% (Figura 6). As retenções determinadas para os dois telhados verdes estão em conformidade às encontradas por Rosseti et al. (2013) e Jr et al. (2014), que apresentaram em seus trabalhos, uma capacidade média de retenção hídrica em telhados verdes entre 39 e 85\%. É importante ressaltar, que a diferença da retenção hídrica entre os TV I e II, pode ser explicada por meio da observação in loco, através da qual foi possível verificar que no TV tipo I ocorreu maior aeração das raízes devido à estrutura da camada de drenagem ter favorecido a presença de alguns espaços vazios. Isso favoreceu o adensamento das raízes com mais $\mathrm{O} 2$ disponível, ocasionando com isso maior absorção de água e sais minerais, resultando em maior crescimento do vegetal e maior interceptação de água pela grama. De acordo com Franco e Inforzato (1946), a boa aeração do solo, favorece o crescimento das raízes, que se subdividem abundantemente, resultando em uma superfície de maior absorção.

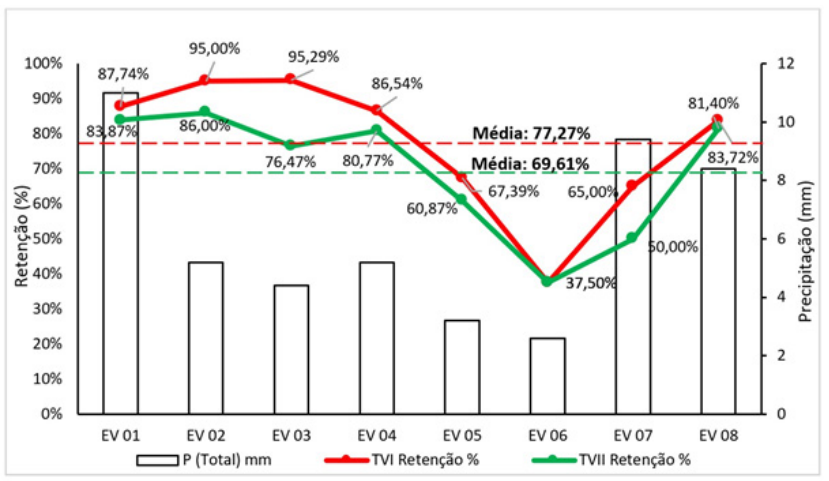

Figura 6 - Taxa percentual de retenção de chuva em cada telhado verde Fonte: Autores
Em relação à redução da vazão de pico de cheia dos telhados verdes extensivos em relação ao telhado convencional, foi observado que a redução de vazão de pico média do TV I foi igual a 85,94\% e a redução de vazão de pico média do TV II foi igual a $80,18 \%$. Estudos realizados no Brasil por Tassi et al. (2014) e Araújo et al. (2014), além de estudos no âmbito internacional (BENGTSSON et al., 2005; CARTER e RASMUSSEN, 2006; VOYDE et al., 2010; FIORETTI et al., 2010; METSELAAR, 2012; STOVIN et al., 2012), indicam que os telhados verdes podem reduzir o escoamento na ordem de $40 \%$ a $89 \%$. Portanto, a redução da vazão de pico apresentada pelos TV I e II mostra-se compatível com a literatura disponível sobre o tema.

\subsection{Custos dos Telhados Verdes}

Na confecção da camada de drenagem do TV I, adotou-se a reutilização de garrafas $\mathrm{PET}$, o que permitiu reduzir o custo total do telhado. Assim, o m2 da camada de drenagem do TV I apresentou custo total de $\mathrm{R} \$ 26,00$. $O$ custo da camada de impermeabilização, camada filtrante, camada de substrato e vegetação tiveram valores de $\mathrm{R} \$$ 54,00; $R \$ 10,00 ; R \$ 35,00$ e $R \$ 7,10$ respectivamente.

A camada de drenagem do TV II, a qual foi pré-fabricada pela empresa ECOTELHADO, apresentou custo total de $\mathrm{R} \$ 82,50$ reais para cada $\mathrm{m} 2$ de área. Logo, o custo da camada filtrante foi de $R \$ 26,40$, além do custo de $R \$ 350,00$ reais de frete cobrado para entrega dos módulos em Belém. A camada de impermeabilização, camada de substrato e de vegetação contabilizaram custos de $R \$ 54,00$, $R \$$ 35,00 e $R \$ 7,10$ reais, respectivamente. Já o custo do telhado convencional foi de $\mathrm{R} \$ 68,00$ reais o metro quadrado.

$\mathrm{Na}$ Tabela 2 verifica-se que o custo do TV I foi menor que o custo do TV II, ou seja, o custo do TV Ié $31,88 \%$ do custo do TV II. Isso mostra que o custo de instalação do TV I é mais viável economicamente devido à adoção de material reciclável no processo de montagem do telhado. Entretanto, não foi contabilizado o custo de montagem desse tipo de telhado verde, o que se trata de uma limitação do presente trabalho. 


\begin{tabular}{|l|l|}
\hline Tipo & Custo $(\mathbf{m} 2)$ \\
\hline TV Tipo I & $\mathrm{R} \$ 197,89$ \\
\hline TV Tipo II & $\mathrm{R} \$ 620,79$ \\
\hline Telhado Convencional & $\mathrm{R} \$ 68,00$ \\
\hline
\end{tabular}

Tabela 2 - Custo total de Instalação

Fonte: Autores

Ainda sobre a Tabela 2, os custos dos TV I e II são bem superiores ao custo do telhado convencional. Isso está de acordo com os trabalhos de Patterson (1998) e Rosseti et al. (2013). Mesmo se considerando o uso de garrafas PET na estrutura do TV I, este ainda apresentou um custo de instalação três vezes superior ao de um telhado convencional. Portanto, ressalta-se, que o alto investimento inicial de um telhado verde extensivo pode ser recuperado via:

- Redução no consumo de energia elétrica, pois o uso de telhados verdes minimiza os efeitos da radiação solar no interior de residências e prédios proporcionando melhor conforto térmico e menor consumo de energia (BEVILACQUA et al., 2016; WILLIAM et al., 2016);

- Redução do efeito de ilha de calor no meio urbano e redução do escoamento superficial, conforme apontado por Karteris et al. (2016) e Razzaghmanesh et al. (2016);

- Uso de garrafas PET, que se justifica por apresentar lenta decomposição no meio ambiente. Pois segundo estudo realizado por Vasconcelos (2007), o tempo de decomposição do politereftalato de etileno (PET) isolado é de 100 anos. E é sabido que o ciclo de vida útil de um teIhado verde dura em média entre 25 e 55 anos e o telhado convencional apenas 20 anos (ACKS, 2006; CLARK et al., 2008; CHAN e CHOW, 2013; e PENG e JIM, 2015). Logo, a adoção da reutilização de garrafas PET na estrutura de um telhado verde não compromete o tempo de vida útil do telhado, pois esse tempo é menor que o tempo mínimo de decomposição de uma garrafa PET. Portanto, a reutilização de garrafas PET em telhados verdes ajuda a retardar o seu descarte no meio ambiente, podendo gerar projetos de créditos de carbono, que compensariam o alto custo inicial dos telhados verdes.

\section{CONCLUSÕES}

O desempenho do TV I foi, na maioria dos eventos de chuva analisados, superior ao desempenho do TV II quanto ao retardo do escoamento superficial. Em relação, a eficiência na capacidade de retenção hídrica entre as estruturas verdes, ficou evidente que o TV I apresenta melhor capacidade de absorção de água em relação ao TV II. Dessa forma, a diferença na capacidade de retenção entre os telhados verdes está diretamente relacionada com a estrutura drenante adotada em cada telhado verde extensivo. A explicação para esse fato está na maior aeração das raízes devido à estrutura da camada de drenagem do TV I possibilitada pelo arranjo dos fundos das garrafas PET. Isso proporcionou a presença de alguns espaços vazios, favorecendo o adensamento das raízes com mais $\mathrm{O} 2$ disponível, ocasionando com isso maior absorção de água e sais minerais, resultando em maior crescimento do vegetal e maior interceptação de água pela grama. Nesse caso, a redução da vazão de pico de cheia dos telhados verdes extensivos variou de $80,18 \%$ a 85,94\% respectivamente (TV II e TV I) comparados ao telhado convencional. Em relação ao custo, o TV I apresentou menor custo de instalação comparado ao TV II. Entretanto, não foi contabilizado o custo de montagem dos telhados verdes, o que se trata de uma limitação do trabalho. Nesse contexto, é possível afirmar que o telhado verde contribui significativamente para a redução de cheias e que o uso de materiais recicláveis na construção de telhados verdes mostra-se uma alternativa viável tanto ambientalmente como economicamente.

\section{AGRADECIMENTOS}

Agradece-se a FAPESPA/CAPES uma bolsa de mestrado e ao CNPq uma bolsa de produtividade em pesquisa através do processo 303542/2018-7.

\section{REFERÊNCIAS}

ACKS, K. A framework of cost-benefit analysis of green roofs: initial estimates, 2006. Disponivel em: http://greenroofs.org/grtok/economic_browse.php?i$d=39 \&$ what=view. Acesso em 09 de Agos. 2016.

ARAÚJO, A. P. C. S et al. Balanço hídrico de sistema modular para telhado verde. Enciclopédia biosfera, Centro Científico Conhecer. Goiânia, v. 10, n. 18, p. 637644, 2014.

BACOVIS, T. M.; NAGALLI, A. Avaliação do desempenho hidrológico de protótipo de telhado verde extensivo. Revista Acadêmica: Ciências Agrárias Ambientais. Curitiba, v. 11, Supl. 1, p. 35-42, 2013.

BENGTSSON, L.; GRAHN, L.; OLSSON, J. Hydrological function of a thin extensive green roof in southern Sweden. Hydrology Research, v. 36, n. 3, p. 259-268, 2005.

BERARDI, U.; HOSEINI, A.; HOSEINI. A. State-of-the-art analysis of the environmental benefits of green roofs. Applied Energy, v. 115, p. 411-428, 2014. BEVILACQUA, P. et al. Experimental investigation of 
the thermal performances of an extensive green roof in the Mediterranean area. Energy and Buildings, v. 122, p. 63-79, 2016.

BIANCHINI, F.; HEWAGE, K. How "green" are the green roofs? Lifecycle analysis of green roof materials. Building and Environment, v. 48, p. 57-65, 2012.

CARTER, T. L.; RASMUSSEN, T. C. Hydrologic Behavior of Vegetated Roofs. Journal of the American Water Resources Association, v. 42, n. 5, p. 1261, 2006.

CHAN, A. L. S.; CHOW, T. T. Energy and economic performance of green roof system under future climatic conditions in Hong Kong. Energy and Buildings, v. 64, p. 182-198, 2013.

CHENANI, S. B.; LEHVÄVIRTA, S.; HÄKKINEN, T. Life cycle assessment of layers of green roofs. Journal of Cleaner Production, v. 90, p. 153-162, 2015.

CLARK, C; ADRIAENS, P; TALBOT, F. B. Green roof valuation: a probabilistic economic analysis of environmental benefits. Environmental science \& technology, v. 42, n. 6, 2008.

COSTA, J.; COSTA, A.; POLETO, C. Telhado Verde: redução e retardo do escoamento superficial. Revista de Estudos Ambientais, v. 14, n. 2, edição especial, p. 50-56, 2012.

CUNHA, S. F. et al. Avaliação da acurácia dos métodos do SCS para cálculo da precipitação efetiva e hidrogramas de cheia. Revista Brasileira de Recursos Hídricos-RBRH, Porto Alegre, v. 20, n. 4, p. 837 - 848, 2015.

FIORETTI, R.; PALLA, A.; LANZA, L G, PRINCIPI, P. Green roof energy and water related performance in the Mediterranean climate. Building and Environment, v. 45, n. 8, p. 1890-1904, 2010.

FRANÇA, L. C. J. O uso do telhado verde como alternativa sustentável aos centros urbanos: opção viável para a sociedade moderna do século XXI. Revista Húmus, v. 2, n. 4, 2012.

FRANCO, C. M.; INFORZATO, R. O sistema radicular do cafeeiro nos principais tipos de solo do estado de São Paulo. Bragantina, Vol. 6, n 9, 1946.

HUANG, Y.; CHEN, C.; TSAI, Y. Reduction of temperatures and temperature fluctuations by hydroponic green roofs in a subtropical urban climate. Energy and Buildings, v. 129, p. 174-185, 2016.

JR, A. A. O; NETO, P. A; MENDIONDO, E. M. Análise da Retenção Hídrica em Telhados Verdes a Partir da Eficiência do Coeficiente de Escoamento. Revista Brasileira de Recursos Hídricos -RBRH, V. 19, n. 2, p. 41-52, 2014.
JUSTINO, E. A.; PAULA, H. M.; PAIVA, E. C. R. Análise do efeito da impermeabilização dos solos urbanos na drenagem de água pluvial do município de UberlândiaMG. Espaço em Revista, v. 13, n. 2, 2012.

KARTERIS, M. et al. Towards a green sustainable strategy for Mediterranean cities: Assessing the benefits of large-scale green roofs implementation in Thessaloniki, Northern Greece, using environmental modelling, GIS and very high spatial resolution remote sensing data. Renewable and Sustainable Energy Reviews, v. 58, p.510-525, 2016.

KÖPPEN, W. et al. Das geographische System der Klimate (Handbuch der Klimatologie, Bd. 1, Teil C).

Borntraeger Science publishers, 1936.

METSELAAR, K. Water retention and evapotranspiration of green roofs and possible natural vegetation types. Resources, conservation and recycling, v. 64, p. 49-55, 2012.

NRCS (2007) National Engineering Handbook, available in ftp://ftp.wcc.nrcs.usda.gov/wntsc/H\&H/ NEHhydrology/ch16.pdf.

NRCS - Natural Resources Conservation Service: US Department of Agriculture (USDA), Urban Hydrology for small watersheds, Technical Release 55 (TR-55), 2nd Edn., Natural Resources Conservation Service, Conservation Engineering Division, USA, 1986.

OHNUMA Jr, A. A., ALMEIDA, N. P. De, \& Mendiondo, E. M. Análise da Retenção Hídrica em Telhados Verdes a Partir da Eficiência do Coeficiente de Escoamento. Revista Brasileira de Recursos Hídricos-RBRH, 19(August), 41-52, 2014.

PATTERSON, M. What color green? Buildings, v. 92, n. 5, p. 80-82, 1998.

PENG, L. L. H.; JIM, C. Y. Economic evaluation of green-roof environmental benefits in the context of climate change: The case of Hong Kong. Urban Forestry \& Urban Greening, v. 14, n. 3, p. 554-561, 2015.

PÉREZ, G. et al. Use of rubber crumbs as drainage layer in green roofs as potential energy improvement material. Applied energy, v. 97, p. 347-354, 2012.

RAZZAGHMANESH, M.; BEECHAM, S.; SALEMI, T. The role of green roofs in mitigating Urban Heat Island effects in the metropolitan area of Adelaide, South Australia. Urban Forestry \& Urban Greening, v. 15, p. 89-102, 2016.

RINCÓN, L. et al. Environmental performance of recycled rubber as drainage layer in extensive green roofs. A comparative Life Cycle Assessment. Building and Environment, v. 74, p. 22-30, 2014. 
ROSSETI, K. A. C. et al. Abordagem sobre as barreiras e benefícios da utilização do sistema de telhado verde em áreas urbanas de regiões tropicais. Brazilian Geographical Journal: Geosciences and Humanities research medium, v. 4, n. 1, p. 55-77, 2013.

SILVA, Dione Galvão da. et al. Crescimento de mudas de hortaliças em substratos orgânicos. Cadernos de Agroecologia - ISSN 2236-7934 - Anais do VI CLAA, X CBA e V SEMDF - Vol. 13, N 1, jul. 2018.

SOUZA, C. F.; CRUZ, M. A. S.; TUCCl, C. E. M. Desenvolvimento Urbano de Baixo Impacto: Planejamento e Tecnologias Verdes para a Sustentabilidade das Águas Urbanas. Revista Brasileira de Recursos Hídricos-RBRH, v. 17, n. 2, p. 9-18, 2012.

STOVIN, V.; VESUVIANO, G.; KASMIN, H. The hydrological performance of a green roof test bed under UK climatic conditions. Journal of Hydrology, v. 414, p. 148-161, 2012.

TARGA, M. S.; BATISTA, G. T.; DINIZ, H. D.; DIAS, N. W.; MATOS, F. C. Urbanização e escoamento superficial na bacia hidrográfica do Igarapé Tucunduba, Belém, PA, Brasil, Revista Ambiente \& Água, v. 7, n.2, p. 120-142, 2012. http://dx.doi.org/10.4136/ambi-agua.905.

TASSI, R. et al. Telhado verde: uma alternativa sustentável para a gestão das águas pluviais. Ambiente Construído, Porto Alegre, v. 14, n. 1, p. 139-154, 2014. TAVARES, J. P. N. Enchentes repentinas na cidade de Belém-PA: condições climáticas associadas e impactos sociais no ano de 1987. Caminhos de Geografia, v. 9, n. 28, p. 1-6, 2009.

TUCCI, C. E. M. Modelos Hidrológicos. 2. Ed. Porto Alegre: ABRH, 2005. 678 p.

VASCONCELOS, Y. Decomposição rápida: pesquisadores desenvolvem polímero reciclado feito com PET inofensivo ao ambiente. Pesquisa FAPESP, n 135, p. 76 - 77, 2007. Disponível em: http://revistapesquisa. fapesp.br/2007/05/01/decomposicao-rapida/. Acesso em 14/03/2019.

VOYDE, E.; FASSMAN, E.; SIMCOCK, R. Hydrology of an extensive living roof under sub-tropical climate conditions in Auckland, New Zealand. Journal of hydrology, v. 394, n. 3, p. 384-395, 2010.

WILLIAM, R. et al. An environmental cost-benefit analysis of alternative green roofing strategies. Ecological Engineering, v. 95, p. 1-9, 2016.

\section{AUTORES}

ORCID: https://orcid.org/0000-0002-3814-1795

EDKEYSE DIAS GONÇALVES | Universidade Federal do Pará | Programa de Pós-Graduação em Engenharia Civil/ITEC/ UFPA | Belém, PARÁ (PA) - Brasil | Rua Augusto Corrêa, 1 Guamá, Belém - PA, 66075-110 | E-mail: edkeyse@ufpa.br

ORCID: https://orcid.org/0000-0001-8022-2647

CLAUDIO JOSÉ CAVALCANTE BLANCO, PhD. | Universidade Federal do Pará | Faculdade de Engenharia Sanitária e Ambiental/ ITEC/UFPA | Belém, PARÁ (PA) - Brasil | Rua Augusto Corrêa, 1 - Guamá, Belém - PA, 66075-110 | E-mail: blanco@ufpa.br

ORCID: https://orcid.org/0000-0002-1057-1398

VANESSA DA ROSA WATRIN, M.Sc.| Universidade Federal do Pará | Programa de Pós-Graduação em Engenharia Civil/ITEC/ UFPA | Belém, PARÁ (PA) - Brasil | Rua Augusto Corrêa, 1 - Guamá, Belém - PA, 66075-110|E-mail: watrin@ufpa.br

ORCID: https://orcid.org/0000-0002-7238-6892

CARLOS EDUARDO AGUIAR DE SOUZA COSTA, M.Sc. | Universidade Federal do Pará | Programa de Pós-Graduação em Engenharia Civil/ITEC/ UFPA | Belém, PARÁ (PA) - Brasil | Rua Augusto Corrêa, 1 - Guamá, Belém - PA, 66075-110| E-mail: eduardoaguiarsc@hotmail.com.

\section{COMO CITAR ESTE ARTIGO}

GONÇALVES, Edkeyse Dias; BLANCO, Claudio José Cavalcante; WATRIN, Vanessa da Rosa; COSTA, Carlos Eduardo Aguiar de Souza. Análise Experimental E Custos De Telhados Verdes Comerciais E Fabricados Com Garrafas PET Para Redução De Cheias Urbanas Na Amazônia. MIX Sustentável, [S.I.], v. 7, n. 2, p. 57-66, abr. 2021. ISSN 24473073. Disponível em:<http://www. nexos.ufsc.br/index.php/mixsustentavel>. Acesso em: dia mês. ano. doi:https://doi.org/10.29183/2447-3073. MIX2021.v7.n2.57-66.

DATA DE ENVIO: 28/09/2020

DATA DE ACEITE: 05/11/2020 
